\section{Intégrité chromosomique et schizophrénie}

Marie-Odile Krebs
Inserm, Laboratoire Physiopathologie de Maladies Psychiatriques (LPMP),

Centre de psychiatrie et Neuroscience, U894 ; Université Paris Descartes,

Service Hospitalo-Universitaire, Hôpital Sainte-Anne,

7, rue Cabanis, 75014 Paris, France. marie-odile.krebs@inserm.fr

\section{Délétions spécifiques} sur les chromosomes 1q21.1, 15q11.2 et $15 q 13.3$ et schizophrénie

Ces premiers résultats ont été confirmés et complétés dans deux articles, parus récemment dans la revue Nature. Le travail coordonné par $\mathrm{H}$. Stefansson [4] concerne l'analyse de 9878 transmissions (analyse de paires parents - enfants) et identifie 66 CNV de novo, dont l'association avec la schizophrénie a été étudiée dans un second groupe de 1433 patients et 33250 «contrôles». Cette population « contrôle » est en réalité une population générale qui contient 299 patients avec un syndrome autistique et vraisemblablement $1 \%$ de patients atteints de schizophrénie. L'analyse a révélé une association de la maladie avec trois délétions situées au niveau du chromosome lq21.1, 15q11.2 et $15 q 13.3$. Ces associations ont été confirmées dans un second groupe de 3285 patients et 7951 contrôles avec un $0 d d$ ratio respectivement de $14,8,2,3$ et 11,5 pour chacune des délétions citées plus haut. Lorsque I'échantillon clinique est restreint à la schizophrénie (exclusion des troubles schizophréniformes, des troubles schizoaffectifs, des troubles délirants...), l'association ne reste significative que pour la délétion en lq21.1.

- Les délétions identifiées en lq21.1 sont de grande taille (1,35 Mb [7]) ou très grande taille $(2,5 \mathrm{Mb}[4]$, incluant la précédente) et identiques chez les contrôles. La forme courte correspond à celle décrite précédemment dans des cas d'autisme, de retard mental ou de schizophrénie. 
- La délétion en $15 q 11.2$ présente chez 26 patients $(0,55 \%)$ et 79 contrôles $(0,19 \%)$ est nettement plus petite $(470 \mathrm{Mb})$. Cette zone est touchée dans certains syndromes d'Angelman ${ }^{2}$ et contient le gène CYFIPl (cytoplasmic FMRI interacting protein 1) qui interagit avec la protéine de I' $X$ fragile (FMRP, fragile $X$ mental retardation protein) pour participer à la régulation de la pousse dendritique et axonale. L'hémizygotie induite par la délétion se traduit par des troubles psychiatriques proches de ceux qui sont observés chez les mères porteuses d'un allèle muté de l'X fragile.

- La délétion en 15q13.3, présente chez 7 patients et 8 contrôles, est de grande taille $(1,53 \mathrm{Mb})$ et inclut le gène codant pour la sous-unité alpha 7 du récepteur nicotinique.

La fréquence des délétions identifiées dans ce travail est globalement comparable à celle qui prévaut en $22 q 11$, présente dans ces populations dans 8 des 3838 cas et aucun des 39299 contrôles.

Les résultats de l'étude de l'International Schizophrenia Consortium [5] sous la coordination de P Sklaar, sont très concordants avec ces observations. Dans cette seconde étude également, les variants structuraux rares couvrant l'ensemble du génome ont été comparés chez 3391 patients et 3181 contrôles. Un total de 6753 CNV a été répertorié. La présence de CNV rares et d'une taille supérieure à $100 \mathrm{~kb}$ est associée à un risque accru de schizophrénie (x 1,15) bien que là encore, des CNV soient présents chez les contrôles (en moyenne $0,99)$. Le nombre de gènes concernés est aussi significativement plus élevé

\footnotetext{
${ }^{2}$ Le syndrome d'Angelman (SA) est une maladie neurogénétique touchant le cerveau, qui se traduit par un ensemble de signes cliniques incluant des troubles du développement moteur (acquisition de la marche, ataxie), un déficit intellectuel avec un langage minimal ou absent, des crises d'épilepsie, des troubles du sommeil, un visage aux traits caractéristiques et un comportement gai avec des rires très faciles. La prévalence de ce syndrome est estimée à l sur 12000 . Le syndrome d'Angelman est lié à la perte de fonction d'un ou plusieurs gènes dans la région $15 q 11-q 13$, soumise à une empreinte parentale (source : orphanet).
}

chez les patients $(x 1,41)$. Cet effet est plus marqué pour les grandes délétions (plus de $500 \mathrm{~kb}$ ), les variants les plus rares (890 CNV uniques) ou ceux impliquant des gènes. Ainsi, la fréquence des délétions de plus de $500 \mathrm{~kb}$ est supérieure de 1,67 chez les patients comparés aux contrôles, et cette différence atteint 3,57 si l'on considère le nombre de gènes touchés. L'effet est plus uniforme pour les duplications, mais les duplications de grandes tailles étaient très peu fréquentes.

Dans cette population, les microdélétions touchant le chromosome 22 ql1.2 correspondaient chez 6 patients à la délétion large $(3 \mathrm{Mb})$; chez 5 à la délétion courte $(1,5 \mathrm{Mb})$ et chez 2 autres patients à des délétions atypiques touchant la partie distale de la délétion de $3 \mathrm{Mb}$ (aucune délétion n'a été détectée chez les contrôles).

En outre, 271 autres délétions de plus $500 \mathrm{~Kb}$ ont été identifiées, et en particulier dans 2 autres régions: $15 q 13.3$ et 1q21.1. Les délétions du chromosome 15 (28-31 Mb) étaient présentes chez 9 patients mais indétectables chez les témoins. Elles sont similaires à celles qui sont décrites par $H$. Stefansson, et incluent une zone récemment identifiée dans certains retards mentaux avec épilepsie [6] contenant le gène codant pour la sous-unité alpha 7 du récepteur nicotinique.

Les régions délétées en lq21.1 (142 à $145 \mathrm{Mb}$ ) décrites par le consortium et par H. Stefansson [4] se superposent, et cette même région a également été identifiée dans les études de liaison [10]; elle est également affectée chez des patients ayant un trouble des apprentissages ou un autisme [7].

Complétant ces observations, Basset et al., [8] ont recherché des CNV sur l'ensemble du génome chez des sujets porteurs de microdélétions $22 q 11.2$, mais n'ont détecté aucun CNV en dehors de la région $22 q 11.2$, confirmant que cette anomalie chromosomique est un facteur de prédisposition majeur pour la schizophrénie.
Que conclure de ces travaux?

Le premier enseignement de ces travaux est d'ordre génétique: ils confortent I'hypothèse selon laquelle quelques mutations, rares, prédisposent à la schizophrénie avec une assez forte pénétrance, et vont à l'encontre de l'hypothèse prévalente des «maladies communes, allèles communs » qui postule que la maladie est liée à une combinaison d'allèles communs, chacun n'ayant qu'un effet modeste. Reste à comprendre la signification des CNV présents dans une proportion non négligeable des sujets contrôles et leurs mécanismes.

Le second enseignement est d'ordre plus physiopathologique : la fréquence de ces variants dans certaines régions chromosomiques spécifiques et dans - ou à proximité de - gènes impliqués dans le neurodéveloppement et la transmission glutamatergique, suggère (ou confirme) ainsi le rôle d'un ensemble de gènes ou de régions candidates. Néanmoins, une modification touchant un gène essentiel au neurodéveloppement a été détectée chez certains témoins, soulignant la prudence dont il faut faire preuve avant d'établir un lien entre un événement unique rare et une pathologie.

Par ailleurs, la duplication de gènes entiers - modifiant le «dosage» de gènes - pourrait aussi avoir des conséquences fonctionnelles, mais ce mécanisme n'a pas été directement étudié dans ces travaux.

\section{Association génotype-phénotype : perspectives cliniques}

Ces travaux apportent par ailleurs un regard nouveau sur la valeur des tableaux cliniques observés, les phénotypes, et sur les liens entre spectres autistique et schizophrénique. II apparaît que les sujets porteurs de variants structuraux se concentrent dans certaines catégories diagnostiques; c'est le cas des formes à début infantile de schizophrénie, pathologie rare, dans lesquelles les variants structuraux sont plus fréquents, les rapprochant ainsi du spectre autistique. II conviendra de déterminer 
si les syndromes schizophréniques associés aux microdélétions identifiées dans l'autisme sont d'authentiques schizophrénies ou s'en distinguent, identifiant de nouveaux syndromes. Néanmoins, à une région chromosomique ou à un gène donné correspond un large spectre phénotypique, y compris l'absence de troubles. Par ailleurs, les patients atteints de troubles schizophréniques apparus après l'âge de 18 ans, considérés comme non précoces, sont déjà porteurs de CNV avec une fréquence accrue ( $>3$ fois) par rapport aux contrôles. Ce pléiotropisme génétique suggère l'influence de facteurs modificateurs, environnementaux ou génétiques.

Loin d'invalider les efforts effectués dans la recherche d' «endophénotypes » (caractérisation des tableaux au-delà des traits les plus cliniquement évidents) permettant d'affiner les phénotypes, ces résultats les encouragent. Néanmoins, il apparaît de plus en plus évident que ces endophénotypes ou phénotypes intermédiaires, doivent être recherchés non plus seulement sur un plan phénoménologique ou cognitif, mais dans une orientation étiologique. Les marqueurs développementaux (anomalies physiques, neurologiques, cérébrales) apparaissent dans ce contexte particulièrement pertinents, plus que ne l'est le simple âge de début qui apparaît peu performant pour distinguer des sous-groupes.

L'idée que des anomalies chromosomiques puissent prédisposer aux troubles schizophréniques n'est pas nouvelle. Néanmoins, ces travaux récents démontrent que le phénomène est beaucoup plus fréquent qu'initialement envisagé et ancrent définitivement la schizophrénie dans le spectre des troubles du neurodéveloppement. En outre, la convergence singulière des résultats des différentes équipes pointe certaines régions $d u$ génome qui sont plus que jamais des régions candidates. $\diamond$

Chromosomal integrity

and schizophrenia

\section{RÉFÉRENCES}

1. Sebat J, Lakshmi B, Malhotra D, et al. Strong association of de novo copy number mutations with autism. Science $2007 ; 316: 445-9$.

2. Walsh T, McClellan JM, McCarthy SE, et al. Rare structural variants disrupt multiple genes in neurodevelopmental pathways in schizophrenia. Science 2008 ; 320 : 539-43.

3. Weiss LA, Shen Y, Korn JM, et al. Autism consortium. Association between microdeletion and microduplication at $16 \mathrm{pl} 1.2$ and autism. $N$ Engl J Med $2008 ; 358: 667-75$

4. Stefansson H, Rujescu D, Cichon S, et al. Large recurrent microdeletions associated with schizophrenia. Nature 2008; 455 : 232-6.

5. International Schizophrenia Consortium. Rare chromosomal deletions and duplications increase risk of schizophrenia. Nature $2008 ; 455$ : 237-41.

6. Sharp AJ, Mefford HC, Li K, et al. A recurrent $15 q 13.3$ microdeletion syndrome associated with mental retardation and seizures. Nat Genet 2008; $40: 322-8$.

7. Mefford HC, Sharp AJ, Baker C, et al. Recurrent rearrangements of chromosome lq21.1 and variable pediatric phenotypes. N Engl J Med 2008 ; 359 : 1685-99.

8. Bassett AS, Marshall CR, Lionel AC, Chow EW, Scherer SW. Copy number variations and risk for schizophrenia in 22ql1.2 deletion syndrome. Hum Mol Genet 2008; $17: 4045-53$.

9. Jamain S, Betancur C, Giros B, Leboyer M, Bourgeron T. La génétique de l'autisme. Med Sci (Paris) 2003 ; 19: 1081-90.

10. Maziade M, Mérette C, Chagnon YC, Roy MA. Génétique de la schizophrénie et de la maladie bipolaire. Med Sci (Paris) $2003 ; 19: 960-6$.

\section{NOUVELLE}

\section{Déterminants cliniques et bactériens au cours des infections extra-intestinales dues à Escherichia coli}

Françoise Jauréguy

\author{
Service de Bactériologie-Virologie-Hygiène, \\ Hôpital Avicenne, AP-HP, UFR Santé, \\ Médecine, Biologie Humaine, Université Paris 13, \\ 125, rue de Stalingrad, 93009 Bobigny Cedex, France. \\ francoise.jaureguy@avc.aphp.fr
}

> Escherichia coli constitue la majeure partie de la flore microbienne commensale aérobie du tube digestif de l'homme; c'est également le bacille à Gram négatif le plus fréquemment responsable d'infections humaines. Celles-ci résultent de la rupture de l'équilibre qui existe entre la bactérie et l'hôte. Le pouvoir pathogène d' $\varepsilon$. coli chez l'homme peut être extrêmement varié. D'un point de vue clinique, on distingue au sein de l'espèce les souches pathogènes intestinales et les souches pathogènes extra-intestinales.
Les souches pathogènes extra-intestinales sont regroupées sous la dénomination commune ExPEC (extraintestinal pathogenic $\varepsilon$. coli) [1]. Les ExPEC sont incapables de produire des infections intestinales, mais peuvent coloniser le tractus intestinal. Ainsi, ce sont des pathogènes opportunistes retrouvés dans les selles des sujets sains avec une fréquence variable selon les individus et les populations humaines étudiées. Les ExPEC peuvent constituer à l'état commensal les souches prédominantes de la flore intestinale chez des hôtes sains (jusqu'à près de $20 \%$ ) [2]. L'acquisition digestive de souches ExPEC par l'hôte ne suffit donc pas à produire une infection, cellesci doivent également avoir accès à un site extra-intestinal. La physiopathologie de l'infection par les ExPEC débute par la colonisation d'une muqueuse et par l'échappement aux systèmes de défenses de l'hôte, et se poursuit par la multiplication dans ce site, voire la dissémination vers d'autres sites, ce qui produit différents dommages chez cet hôte. 